\title{
Neuropeptide Y improves cisplatin-induced bone marrow dysfunction without blocking chemotherapeutic efficacy in
} a cancer mouse model

\author{
Min Hee Park, 1,2,,\# In Kyung Jung ${ }^{1,2,3, \#}$, Woo-Kie Min ${ }^{4}$, Jin Ho Choi ${ }^{5}$, Gyu Man Kim ${ }^{6}$, Hee Kyung Jin ${ }^{1,7, *} \mathcal{E}^{2}$ \\ Jae-sung Bae $e^{1,2,3, *}$ \\ ${ }^{1}$ Stem Cell Neuroplasticity Research Group, Kyungpook National University, Daegu 41566, ${ }^{2}$ Department of Physiology, Cell and Matrix \\ Research Institute, School of Medicine, Kyungpook National University, Daegu 41944, ${ }^{3}$ Department of Biomedical Science, BK21 Plus \\ KNU Biomedical Convergence Program, Kyungpook National University, Daegu 41944, ${ }^{4}$ Department of Orthopaedic Surgery, Kyungpook \\ National University Hospital, Daegu 41944, ${ }^{5}$ Department of Mechanical Engineering, Gumi University, Gumi 39213, ${ }^{6}$ School of Mechanical \\ Engineering, Kyungpook National University, Daegu 41566, ${ }^{7}$ Department of Laboratory Animal Medicine, College of Veterinary Medicine, \\ Kyungpook National University, Daegu 41566, Korea
}

Cisplatin is the most effective and widely used chemotherapeutic agent for many types of cancer. Unfortunately, its clinical use is limited by its adverse effects, notably bone marrow suppression leading to abnormal hematopoiesis. We previously revealed that neuropeptide $\mathrm{Y}$ (NPY) is responsible for the maintenance of hematopoietic stem cell (HSC) function by protecting the sympathetic nervous system (SNS) fibers survival from chemotherapy-induced bone marrow impairment. Here, we show the NPY-mediated protective effect against bone marrow dysfunction due to cisplatin in an ovarian cancer mouse model. During chemotherapy, NPY mitigates reduction in HSC abundance and destruction of SNS fibers in the bone marrow without blocking the anticancer efficacy of cisplatin, and it results in the restoration of blood cells and amelioration of sensory neuropathy. Therefore, these results suggest that NPY can be used as a potentially effective agent to improve bone marrow dysfunction during cisplatinbased cancer therapy. [BMB Reports 2017; 50(8): 417-422]

\section{INTRODUCTION}

The majority of cancer therapies are based on chemotherapeutic agents with cytotoxic effects, which cause cancer cell

*Corresponding authors. Jae-sung Bae, Tel: +82-53-420-4815; Fax: +82-53-424-3349; E-mail: jsbae@knu.ac.kr, Hee Kyung Jin, Tel: +82-53-950-5966; Fax: +82-53-950-5955; E-mail: hkjin@knu.ac.kr ${ }^{\text {\#} T h e s e ~ a u t h o r s ~ c o n t r i b u t e d ~ e q u a l l y ~ t o ~ t h i s ~ w o r k . ~}$

https://doi.org/10.5483/BMBRep.2017.50.8.099

Received 13 June 2017, Revised 29 June 2017, Accepted 7 July 2017

Keywords: Bone marrow dysfunction, Cancer, Chemotherapy-induced side effects, Hematopoietic stem cell, Neuropeptide Y death by directly damaging DNA or by inhibiting cell division. Unfortunately, these agents are non-specific, thus, their administration often induces extended toxic effects in normal tissue as well (1). Cisplatin, which is one of the most widely used chemotherapeutic drugs (2-5), has been employed for the treatment of solid cancers such as ovarian, testicular, uterine, breast, stomach, brain, head-neck, and lung cancer (6-9). Although cisplatin has potent anticancer effects, its use is limited by various side effects such as neurotoxicity, nephrotoxicity, ototoxicity, and particularly bone marrow suppression (4, 10-13). Cisplatin-induced bone marrow damage is accompanied by acute nerve injury in the bone marrow $(\mathrm{BM})$, resulting in sensory and autonomic neuropathy. Patients that have previously received cisplatin show irreversible chronic bone marrow failure, leading to the impairment of hematopoietic stem cells (HSCs) and bone marrow regeneration (13-15). Therefore, it is important to prevent bone marrow dysfunction during conventional chemotherapy using cisplatin without diminishing its anticancer efficacy.

Neuropeptide $Y$ (NPY) is secreted from the brain or sympathetic nerves in the autonomic system and its involvement in a variety of physiological processes, including food intake, energy storage, anxiety, stress, and pain perception, is well known (16-18). Several studies have reported that NPY is implicated in the regulation of cell death processes (19). Particularly, our recent study demonstrated that NPY can prevent sensory neuropathy and reduction in HSC abundance by protecting sympathetic nervous system (SNS) fibers in cisplatin-treated mice, suggesting the therapeutic potential of NPY to improve chemotherapy-induced bone marrow suppression $(20,21)$.

In this study, we have indeed demonstrated the protective effect of NPY against cisplatin-induced bone marrow dysfunction in a mouse model of ovarian cancer. Moreover, we found that NPY did not influence the chemotherapeutic effects of

ISSN: 1976-670X (electronic edition)

Copyright (c) 2017 by the The Korean Society for Biochemistry and Molecular Biology

(c) This is an open-access article distributed under the terms of the Creative Commons Attribution Non-Commercial License (http://creativecommons.org/licenses/by-nc/4.0) which permits unrestricted non-commercial use, distribution, and reproduction in any medium, provided the original work is properly cited. 
cisplatin, while protecting against reduction in HSC abundance and nerve injury from bone marrow impairment, suggesting its potential clinical utility as a protective agent for patients treated with chemotherapy.

\section{RESULTS}

\section{NPY does not affect the anticancer efficacy of cisplatin in an} ovarian cancer mouse model

Prior to determining the mitigating effect of NPY on cisplatininduced bone marrow damage in a mouse model of cancer, we first tested whether NPY has influence on the anticancer efficacy of cisplatin. To establish a cancer xenograft mouse model, A2780 human ovarian cancer cells were transplanted subcutaneously in female athymic nude mice. After cancer establishment, the mice were randomized into 3 groups. One group was subjected to intraperitoneal injection (i.p.) with 10 $\mathrm{mg} / \mathrm{kg}$ cisplatin, the second group was treated with $10 \mathrm{mg} / \mathrm{kg}$ cisplatin plus $50 \mu \mathrm{g} / \mathrm{kg} \mathrm{NPY}$, and the control group was treated with phosphate-buffered saline (PBS); the treatment duration was 7 weeks (Fig. 1A). During the observation period of 7 weeks, the PBS-treated group showed continuous increase in body weight and tumor growth, whereas the cisplatin-alone or

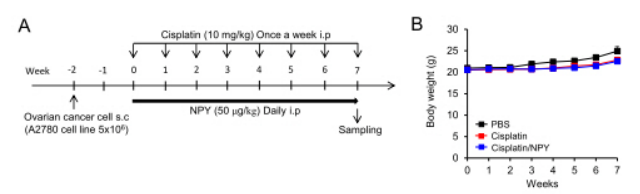

C
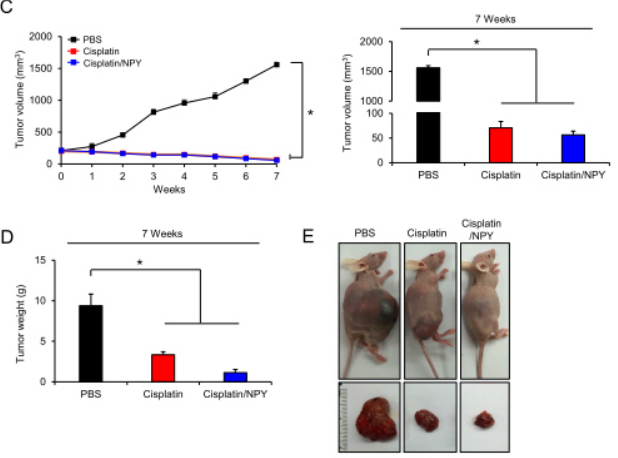

Fig. 1. Influence of NPY on the therapeutic efficacy of cisplatin in a mouse ovarian cancer model. (A) Experimental design to investigate the effect of NPY in cisplatin-induced bone marrow dysfunction. A cancer mouse model was established in athymic nude mice by inoculation of A2780 ovarian cancer cells. After the tumors had grown to approximately $200 \mathrm{~mm}^{3}$, the animals were randomly divided into 3 groups, which were treated with PBS (daily), $10 \mathrm{mg} / \mathrm{kg}$ cisplatin (once a week), or $10 \mathrm{mg} / \mathrm{kg}$ cisplatin (once a week) plus $50 \mu \mathrm{g} / \mathrm{kg}$ NPY (daily). (B) Body weight and (C) tumor volume of each group during treatment (left) or after 7 weeks (right) ( $n=5$ mice per group). (D) Tumor weight after 7 weeks. ( $\mathrm{n}=5$ mice per group). (E) Representative mice and dissected tumors. ${ }^{*} P<0.05$. All error bars indicate the standard errors of the mean (S.E.M.). cisplatin plus NPY-treated groups did not show any gain in weight and tumor volume (Fig. 1B, C). At the end of 7 weeks, the average tumor weights were $9.38 \mathrm{~g}$ in the PBS group, 3.34 $\mathrm{g}$ in the cisplatin group, and $1.12 \mathrm{~g}$ in the cisplatin-plus-NPY group (Fig. 1D, E). Interestingly, the cisplatin plus NPY-treated group showed a tendency for further decrease in tumor weight. Therefore, these results suggest that NPY did not affect the anticancer efficacy of cisplatin therapy in an ovarian cancer mouse model.

\section{NPY prevents cisplatin-induced reduction of HSC abundance in BM}

Decrease in the number of HSCs, which are responsible for the regeneration and repopulation of all blood cell lineages, is a main pathogenesis mechanism in cisplatin-induced bone marrow dysfunction $(13,15,22)$. To examine whether NPY could prevent reduction in HSC abundance upon cisplatin treatment in our ovarian cancer model, we analyzed phenotypic HSCs in BM by flow cytometry. Although no significant differences in the numbers of bone marrow nucleated cells (BMNCs) were observed between the groups (Fig. 2A), the PBS- and cisplatin-treated groups in the cancer-induced groups showed a marked decrease in the number of $\mathrm{Lin}^{-} \mathrm{Sca} 1^{+} \mathrm{C}_{-} \mathrm{Kit}^{+}$(LSK) and $\mathrm{LSKCD}^{+} 8^{-} \mathrm{CD} 150^{+}$ (LT-HSCs) cells compared to the PBS-treated sham group. However, cisplatin plus NPY-treated group in the cancerinduced groups showed increase of BM HSCs as normal condition (PBS-treated sham group) (Fig. 2B-D). These results implied that NPY treatment recovered reduction of BM HSCs, and promoted its survival. Moreover, NPY prevented the reduction of blood cell lineages observed in the cisplatin-
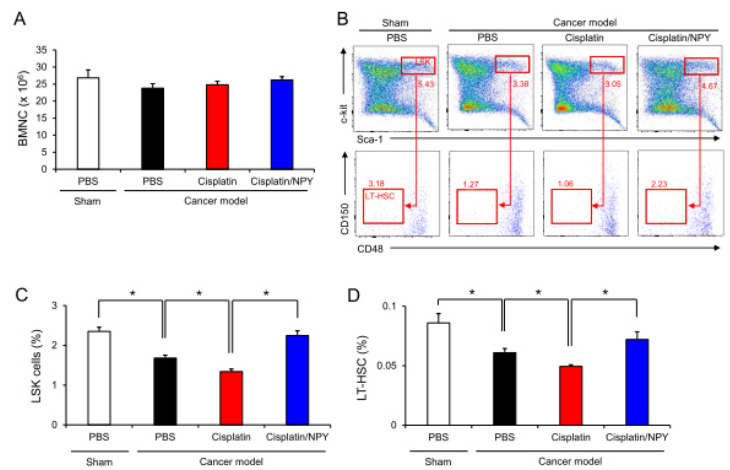

Fig. 2. Mitigation of cisplatin-induced reduction in HSC abundance by NPY treatment. (A) Number of BMNCs of each group after 7 weeks of treatment ( $n=5$ mice per group). (B) Representative flow cytometry plot from the BM of each group ( $n=3-4$ mice per group). The BM was gated first based on Lineage ${ }^{-}$cells, then based on Sca- ${ }^{+}$c-kit $^{+}$to detect LSK cells, and finally based on $\mathrm{CD}^{-}{ }^{-} \mathrm{CD}_{150}{ }^{+}$cells to detect LT-HSCs. (C and D) Percentage of LSK (Lineage ${ }^{-} \mathrm{Sca}^{+}{ }^{+} \mathrm{C}^{-k i t^{+}}{ }^{+}$cells and LT-HSCs (Lineage Sca- ${ }^{+}$c-kit $^{+} \mathrm{CD}^{-} 8^{-} \mathrm{CD}_{150}{ }^{+}$) in the BM of each group ( $\mathrm{n}=$ 3-4 mice per group). ${ }^{*} P<0.05$. All error bars indicate S.E.M. 
treated group (Table 1). Taken together, these results suggest that NPY could ameliorate cisplatin-induced HSC impairment in an ovarian cancer mouse model.

\section{NPY counteracts cisplatin-induced reduction of SNS fiber and EC abundance in the BM}

Recent studies reported that cisplatin-induced SNS injury in BM induces sensory neuropathy, and impairs HSC function by reducing the number of endothelial cells (ECs), which are cells of the BM microenvironment cell related to HSC survival (15, 23). In addition, our previous study demonstrated that NPY is required for the maintenance of HSC function by protecting SNS fibers and EC survival within the BM $(20,21)$. Here, we confirmed these protective roles of NPY during chemotherapy in an ovarian cancer model, where cisplatin-induced sensory neuropathy was ameliorated by NPY treatment (Fig. 3A). The decrease in the number of SNS fibers, which were stained with an antibody against the catecholaminergic enzyme tyrosine hydroxylase (Th), and of ECs was also prevented in the NPY-treated group compared to that by cisplatin alone (Fig. 3B, C). The cytotoxic effects of cisplatin induce direct cell death in cancers and even in normal tissues, and NPY can protect against cell death by regulating apoptosis signaling (19-21). We observed increased apoptosis levels in the BM of PBS- or cisplatin-treated groups, whereas the cisplatin plus NPY-treated group showed significant reduction in apoptosis (Fig. 3D). Taken together, these results strongly suggest that NPY can prevent sensory neuropathy and bone marrow damage during cisplatin therapy in an ovarian cancer model.

\section{DISCUSSION}

Chemotherapy-induced BM suppression is a severe side effect of cancer therapy. Moreover, in cancer patients, chronic BM damage due to chemotherapy is accompanied by impaired HSC function and mobilization, and leads to hematopoiesis
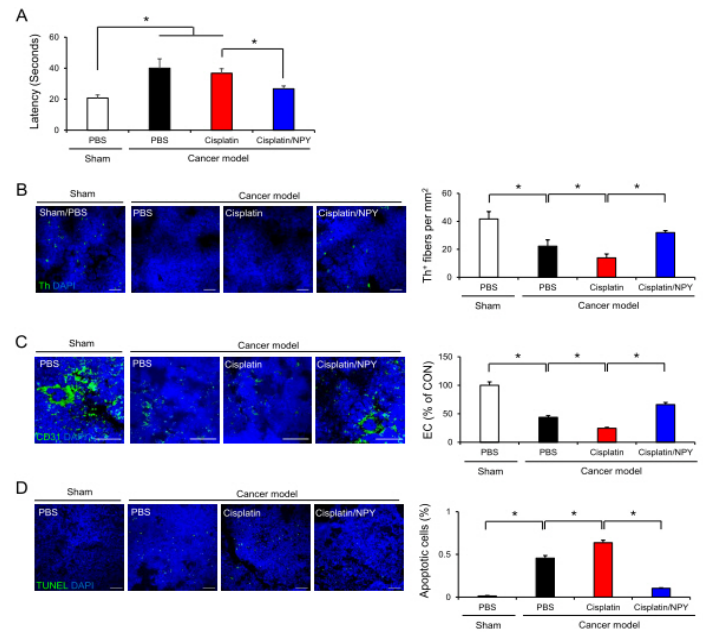

Fig. 3. Protective effect of NPY against cisplatin-induced sensory neuropathy and cell death in the BM microenvironment. (A) Quantification of sensory neuropathy in each group $(n=5$ mice per group). (B) Left, representative immunofluorescence images to detect the presence of $\mathrm{Th}^{+}$fibers. Scale bar, $50 \mu \mathrm{m}$. Right, quantification of $\mathrm{Th}^{+}$fibers in the BM of each group ( $\mathrm{n}=5$ mice per group). (C) Left, representative immunofluorescence BM images of $\mathrm{CD}_{31}{ }^{+}$ECs. Scale bar, $40 \mu \mathrm{m}$. Right, number of $\mathrm{CD} 31^{+}$ECs per femur in each group ( $\mathrm{n}=5$ mice per group). (D) Left, representative immunofluorescence images of the BM showing apoptosis by TUNEL staining. Scale bar, $50 \mu \mathrm{m}$. Right, percentage of apoptotic cells in BM of each group $(n=5$ mice per group). ${ }^{*} \mathrm{P}<0.05$. All error bars indicate S.E.M.

Table 1. Increased blood cell recovery in NPY-treated mice models of cancer with cisplatin treatment. Complete blood counts ofeach group $(\mathrm{n}=5$ mice per group)

\begin{tabular}{|c|c|c|c|c|}
\hline & \multirow{2}{*}{$\begin{array}{c}\text { Sham } \\
\text { PBS }\end{array}$} & \multicolumn{3}{|c|}{ Cancer } \\
\hline & & PBS & Cisplatin & Cisplatin/NPY \\
\hline WBC (K/ml) & $0.9 \pm 0.1$ & $0.3 \pm 0.03 *$ & $0.5 \pm 0.1^{*}$ & $0.8 \pm 0.1^{\#}$ \\
\hline $\mathrm{RBC}(\mathrm{M} / \mathrm{ml})$ & $8.7 \pm 0.2$ & $5.5 \pm 0.2^{*}$ & $6.8 \pm 0.7^{*}$ & $8.3 \pm 0.2^{\#}$ \\
\hline Hgb (g/dl) & $14.7 \pm 0.2$ & $10.8 \pm 0.5$ & $14.5 \pm 0.2$ & $14.2 \pm 0.1$ \\
\hline НCТ (\%) & $75.0 \pm 0.8$ & $58.0 \pm 3.1^{*}$ & $69.7 \pm 3.5$ & $71.5 \pm 0.6$ \\
\hline MCV (fl) & $86.1 \pm 1.1$ & $113.6 \pm 3.0$ & $85.9 \pm 0.8$ & $89.5 \pm 4.0$ \\
\hline $\mathrm{MCH}(\mathrm{pg})$ & $16.8 \pm 0.2$ & $21.2 \pm 0.8$ & $17.9 \pm 0.9$ & $17.4 \pm 0.5$ \\
\hline MCHC (g/dl) & $19.6 \pm 0.1$ & $18.6 \pm 0.2$ & $19.7 \pm 0.2$ & $19.5 \pm 0.3$ \\
\hline RDW-CV (\%) & $19.0 \pm 0.4$ & $26.4 \pm 2.8$ & $20.3 \pm 1.4$ & $18.7 \pm 0.6$ \\
\hline $\operatorname{PLT}(\mathrm{K} / \mathrm{ml})$ & $1002.8 \pm 18.2$ & $895.5 \pm 63.1$ & $856.5 \pm 79.8$ & $946.6 \pm 36.0^{\#}$ \\
\hline
\end{tabular}

WBC: white blood cells, RBC: red blood cells, Hgb: hemoglobin, HCT: hematocrit, MCV: mean cell volume, $\mathrm{MCH}$ : mean corpuscular hemoglobin content, MCHC: mean corpuscular hemoglobin concentration, RDW-CV: red cell distribution width- coefficient of variation, PLT: platelets, MPV: mean platelet volume. ${ }^{*} \mathrm{P}<0.05$ versus Sham/PBS group. ${ }^{\#} \mathrm{P}<0.05$ versus Cancer/Cisplatin group. 
abnormalities (13, 14, 24, 25). Neurotoxicity due to chemotherapy drugs such as cisplatin can damage autonomic nerves in the $\mathrm{BM}$ and compromise hematopoietic regeneration, suggesting that neuroprotection may preserve hematopoietic reserves and regulate HSC mobility after chemotherapy (15). Recently, we demonstrated new roles of NPY as a regulator of HSC survival and mobilization $(20,21,26)$. In particular, we revealed that NPY treatment prevented cisplatin-induced HSC impairment and reduced cisplatin-induced apoptosis of SNS fibers and cells of the BM microenvironment, showing that the neuroprotective effects of NPY could mitigate HSC dysfunction due to chemotherapy $(20,21)$. However, the protective effects of NPY in BM has not been fully explored during cisplatin therapy in cancer mouse models.

In this study, we first tested whether NPY diminished the chemotherapeutic effects of cisplatin in any ovarian cancer mouse model. The results show that NPY did not inhibit the anticancer efficacy of cisplatin; instead, the decrease in the tumor weight upon cisplatin treatment was more pronounced in the presence of NPY, suggesting that NPY might enhance the anticancer efficacy of cisplatin (Fig. 1). In addition, we observed that NPY treatment prevented the cisplatin-induced HSC suppression, resulting in the recovery of the complete blood count (Fig. 2). This is important because NPY can be administered in patients who either have undergone chemotherapy or suffer from disorders with abnormal hematopoiesis. The sympathetic nerve fibers regulate HSC survival and trafficking by acting on cells of the BM niche, and destruction of nerve fibers in the BM causes critical peripheral neuropathies (13-15). Our results show that decrease in Th fiber abundance by cisplatin in an ovarian cancer model was prevented by NPY treatment, leading to the mitigation of sensory neuropathy. Moreover, NPY significantly counteracted the cisplatin-induced reduction in EC abundance and increased apoptosis levels in the BM (Fig. 3). Taken together, these observations indicate that in an ovarian cancer mouse model, NPY could protect against sensory neuropathy and BM damage without blocking the chemotherapeutic efficacy of cisplatin.

Nephrotoxicity, which is another side effect of chemotherapy, also limits the use of cisplatin in cancer therapy. We recently confirmed that NPY treatment could attenuate cisplatin-induced nephrotoxicity by regulating the pro-apoptotic pathway, resulting in protection against renal dysfunction (27). This our previous study suggests that NPY can also be used as an effective agent for renoprotection, as well as neuroprotection. During the last few years, several researchers have demonstrated that a broad range of natural compounds may mitigate the side effects of chemotherapy. Importantly, NPY is a stable peptide that is naturally synthesized in the human body. Therefore, therapeutic applications using NPY may provide clinical benefits and reduce side effects in patients previously treated with chemotherapy.

\section{MATERIALS AND METHODS}

\section{Ovarian cancer xenograft mouse model}

Six- to 8-week-old, female, athymic nude mice (BALB/c Slc-nu/nu) were purchased from SLC (Japan). The A2780 cells were obtained from ECACC (93112519, Salisbury, Wiltshire/UK) and cultivated in RPMI-1640 medium supplemented with 10\% FBS, and $1 \%$ streptomycin and penicillin (all from Gibco). Cells were grown at $37^{\circ} \mathrm{C}$ in a humidified atmosphere containing $5 \% \quad \mathrm{CO}_{2}$. Female athymic nude mice were inoculated with $5 \times 10^{6} \mathrm{~A} 2780$ cells in $100 \mu \mathrm{l}$ saline by a subcutaneous injection at the flank. After inoculation, tumor growth was monitored for approximately 2 weeks, using a vernier caliper. When the tumor size reached approximately $200 \mathrm{~mm}^{3}$, the animals were randomly divided into 3 groups to receive a 7-week treatment with cisplatin, cisplatin plus NPY, or PBS as a control. During the treatment, body weight and tumor size were monitored once a week. A block randomization method was used to divide the animals into different experimental groups. To eliminate bias, investigators were blinded during data collection and analysis. Mice were housed under a 12-hour day-night cycle with free access to tap water and food pellets. All mouse studies were approved by the Kyungpook National University Institutional Animal Care and Use Committee.

\section{Drug treatment}

Cisplatin (Enzo; $10 \mathrm{mg}$ per kg of body weight, once per week) was used for chemotherapy, and the mice received i.p. injections of cisplatin for 7 weeks. To investigate the protective effect of NPY against cisplatin-induced BM dysfunction, the mice received i.p. injections of NPY (Bachem; $50 \mu \mathrm{g}$ per kg body weight, $\mathrm{H}$-6375) daily during the 7-week cisplatintreatment period. After $1 \mathrm{~h}$ from the last injection, the BM and blood were collected and analyzed.

\section{Flow cytometry}

The $\mathrm{BM}$ was flushed from the tibia and femur of each mouse. Red blood cells (RBCs) were lysed once for $5 \mathrm{~min}$ at $4^{\circ} \mathrm{C}$ in $0.15 \mathrm{M} \mathrm{NH}_{4} \mathrm{Cl}$ (StemCell Technologies), washed once with PBS (Gibco), and counted using a cell counter (LUNA ${ }^{\text {TM }}$ Automated Cell Counter). For the detection of LSK cells and LT-HSCs, Lineage ${ }^{+}$cells were removed by magnetic depletion using biotinylated lineage-specific antibodies (CD5, CD45R, CD11b, Gr-1, and Ter-119), followed by depletion with MACs beads conjugated to a monoclonal anti-biotin (Miltenyi Biotec). Lineage ${ }^{-}$cells were stained with phycoerythrin PECy7-conjugated antibodies to Sca1 (558162), APC-conjugated antibodies to c-kit (553356), FITC-conjugated antibodies to CD48 (557484), and PE-conjugated antibodies to CD150 (561540), all from BD Biosciences. Cells were further stained with streptavidin-pacific blue (PB) (Invitrogen, S11222). Data were collected on a BD AriallI (BD Biosciences) and analyzed using the Flowjo software (Tree Star). 
Quantification of sensory neuropathy by the heated-pad assay To evaluate the effects of different treatments on the sensory response, we performed the hot-plate test, as previously described (28). We used a flattering hot plate (Panlab, Harvard Apparatus) maintained at $50^{\circ} \mathrm{C}$. Mice were individually placed on top of the heated surface, and the time to the first episode of nociception (jumping or paw licking) was measured. The cutoff time was $60 \mathrm{~s}$. Between measurements, the heated surface was thoroughly cleaned with detergent and ethanol, and the temperature was allowed to stabilize to $50^{\circ} \mathrm{C}$.

\section{Immunofluorescence staining of BM sections}

Frozen BM sections were prepared and immunostained according to a previously published method (29). The BM sections were fixed in dry ice/hexane, and incubated first with the primary antibody and then with a secondary antibody conjugated with Alexa488 (Life Technologies). Immunofluorescence data were obtained and analyzed using a laser scanning confocal microscope equipped with the Fluoview SV1000 imaging software (Olympus FV1000; Japan). The MetaMorph software (Molecular Devices) was used to calculate the average intensity and cell number. Antibodies used were as follows: Th (Millipore, AB152 or AB318, 1:250 dilution), and CD31 (BD Biosciences, 550300, 1:50 dilution). TUNEL assays were performed using the In Situ Cell Detection Kit, Fluorescein (Roche Diagnostic) according to the manufacturer's instructions.

\section{Statistical analysis}

One way analysis of variance (ANOVA) was conducted, followed by Tukey's HSD test for comparisons between more than two groups. All statistical analyses were performed using the SPSS statistical software. A value of $P<0.05$ was considered to denote statistical significance.

\section{ACKNOWLEDGEMENTS}

This research was supported by the Bio \& Medical Technology Development Program (2017M3A9B4030782) and Basic Science Research Program (2016R1D1A1B03934526) of the NRF funded by the Korean government (MSIP).

\section{CONFLICTS OF INTEREST}

The authors have no conflicting financial interests.

\section{REFERENCES}

1. Blanchard EM (2012) Cisplatin and solid tumours: still working, after all these years. J Solid Tumors 2, 26-33

2. Siddik ZH (2003) Cisplatin: mode of cytotoxic action and molecular basis of resistance. Oncogene 22, 7265-7279

3. Wang D and Lippard SJ (2005) Cellular processing of platinum anticancer drugs. Nat Rev Drug Discov 4,
307-320

4. Cepeda V, Fuertes MA, Castilla J, Alonso C, Queve-do C and Perez JM (2007) Biochemical mechanisms of cisplatin cytotoxicity. Anticancer Agents Med Chem 7, 3-18

5. Einhorn LH (2002) Curing metastatic testicular cancer. Proc Natl Acad Sci U S A 99, 4592-4595

6. Armstrong DK, Bookman MA, McGuire W, Bristow RE, Schilder JM and Group GO (2007) A phase I study of paclitaxel, topotecan, cisplatin and filgrastim in patients with newly diagnosed advanced ovarian epithelial malignancies: a Gynecologic Oncology Group study. Gynecol Oncol 105, 667-671

7. Ridwelski K, Gebauer T, Fahlke J et al (2001) Combination chemotherapy with docetaxel and cisplatin for locally advanced and metastatic gastric cancer. Ann Oncol 12, 47-51

8. Cullen KJ, Yang Z, Schumaker L and Guo Z (2007) Mitochondria as a critical target of the chemotheraputic agent cisplatin in head and neck cancer. J Bioenerg Biomembr 39, 43-50

9. Niell HB, Herndon JE, Miller AA et al (2005) Randomized phase III intergroup trial of etoposide and cisplatin with or without paclitaxel and granulocyte colony-stimulating factor in patients with extensive-stage small-cell lung cancer: Cancer and Leukemia Group B Trial 9732. J Clin Oncol 23, 3752-3759

10. Chaudhry V, Rowinsky EK, Sartorius SE, Donehower RC and Cornblath DR (1994) Peripheral neuropathy from taxol and cisplatin combination chemotherapy: clinical and electrophysiological studies. Ann Neurol 35, 304-311

11. Pabla N, Dong G, Jiang M et al (2011) Inhibition of PKC $\delta$ reduces cisplatin-induced nephrotoxicity without blocking chemotherapeutic efficacy in mouse models of cancer. J Clin Invest 121, 2709-2722

12. Hatzopoulos S, Di Stefano M, Albertin A and Martini A (1999) Evaluation of cisplatin ototoxicity in a rat animal model. Ann NY Acad Sci 884, 211-225

13. Noach EJ, Ausema A, van Os R et al (2000) Chemotherapy prior to autologous bone marrow transplantation impairs long-term engraftment in mice. Exp Hematol 28, 1325-1333

14. Banfi A, Podestà M, Fazzuoli L et al (2001) High-dose chemotherapy shows a dose-dependent toxicity to bone marrow osteoprogenitors: a mechanism for post-bone marrow transplantation osteopenia. Cancer 92, 24192428

15. Lucas D, Scheiermann C, Chow A et al (2013) Chemotherapy-induced bone marrow nerve injury impairs hematopoietic regeneration. Nat Med 19, 695-703

16. Zukowska-Grojec Z (1995) Neuropeptide Y. A novel sympathetic stress hormone and more. Ann NY Acad Sci $771,219-233$

17. Kalra SP and Kalra PS (2004) NPY and cohorts in regulation appetite, obesity and metabolic syndrome: beneficial effects of gene therapy. Neuropeptides 38, 201-211

18. Kuo LE, Kitlinska JB, Tilan JU et al (2007) Neuropeptide Y acts directly in the periphery on fat tissue and mediates stress-induced obesity and metabolic syndrome. Nat Med $13,803-811$ 
19. Santos-Carvalho A, Elvas F, Alvaro AR, Ambrósio AF and Cavadas C (2013) Neuropeptide $Y$ receptors activation protects rat retinal neural cells against necrotic and apoptotic cell death induced by glutamate. Cell Death Dis 4, e636

20. Park MH, Jin HK, Min WK et al (2015) Neuropeptide $Y$ regulates the hematopoietic stem cell microenvironment and prevents nerve injury in the bone marrow. EMBO J 34, 1648-1660

21. Park MH, Min WK, Jin HK and Bae JS (2015) Role of neuropeptide $Y$ in the bone marrow hematopoietic stem cell microenvironment. BMB Rep 48, 645-646

22. Can A (2008) Haematopoietic stem cells niches: Interrelations between structure and function. Transfus Apher Sci 38, 261-268

23. Butler JM, Nolan DJ, Vertes EL et al (2010) Endothelial cells are essential for the self-renewal and repopulation of Notch-dependent hematopoietic stem cells. Cell Stem Cell 6, 251-264

24. Robinson SN, Freedman AS, Neuberg DS, Nadler LM and Mauch PM (2000) Loss of marrow reserve from dose-intensified chemotherapy results in impaired hematopoietic reconstitution after autologous transplantation: $\mathrm{CD} 34+, \mathrm{CD} 34+38-$ and week-6 CAFC assays predict poor engraftment. Exp Hematol 28, 1325-1333

25. Perseghin P, Terruzzi E, Dassi M et al (2009) Management of poor peripheral blood stem cell mobilization: incidence, predictive factors, alternative strategies and outcome. A retrospective analysis on 2177 patients from three major Italian institutions. Transfus Apher Sci 41, 33-37

26. Park MH, Lee JK, Kim N et al (2016) Neuropeptide $\mathrm{Y}$ Induces Hematopoietic Stem/Progenitor Cell Mobilization by Regulating Matrix Metalloproteinase-9 Activity through Y1 Receptor in Osteoblasts. Stem Cells 34, 2145-2156

27. Kim N, Min WK, Park MH, Lee JK, Jin HK and Bae JS (2016) Neuropeptide Y protects kidney against cisplatininduced nephrotoxicity by regulating p53-dependent apoptosis pathway. BMB Rep 49, 288-292.

28. Aloe L, Manni L, Properzi F, De Santis S and Fiore $M$ (2000) Evidence that nerve growth factor promotes the recovery of peripheral neuropathy induced in mice by cisplatin: behavioral, structural and biochemical analysis. Auton Neurosci 86, 84-93

29. Kawamoto $T$ (2003) Use of a new adhesive film for the preparation of multipurpose fresh-frozen sections from hard tissues, whole-animals, insects and plants. Arch Histol Cytol 66, 123-143 